PROCEEDINGS OF THE

AMERICAN MATHEMATICAL SOCIETY

Volume 124, Number 12, December 1996, Pages 3699-3702

S 0002-9939(96)03404-1

\title{
DANES' DROP THEOREM IN LOCALLY CONVEX SPACES
}

\author{
CHENG LIXIN, ZHOU YUNCHI, AND ZHANG FONG
}

(Communicated by Palle E. T. Jorgensen)

Abstract. Danes' Drop Theorem is generalized to locally convex spaces.

\section{INTRODUCTION}

Suppose that $(E,\|\cdot\|)$ is a Banach space and $B$ is the closed unit ball of $E$. By a drop $D(x, B)$ determined by a point $x$ with $\|x\|>1$, we shall mean the convex hull of the set $\{x\} \cup B$. If a nonempty closed set $A$ of Banach space $E$ with a positive distance from the unit ball $B$ is given, then there exists $a \in A$ such that $D(a, B) \cap A=\{a\}$, which is the so-called Danes' Drop Theorem [1]. The drop theorem has found many applications in various situations (see, for instance, [2], [5], [6], [7], [8] and [9]) and it is equivalent to Ekeland's variational principle.

Modifying the concept underlying Danes' Drop Theorem, Rolewicz [3] and Giles and Kutzarova [4] introduced the notion of the drop and weak drop properties and many papers have appeared (see, for instance, [10], [11], [12] and [15]).

This note generalizes Danes' Drop Theorem to locally convex spaces and it is done by substituting "sequentially closed bounded convex set $C$ " in the space for "the closed unit ball $B$ " of the Banach space and " $A$ is strongly Minkowski separated from $C$ " for " $A$ is a positive distance from $B$ ".

\section{Minkowski SePARAtion OF SETS}

Definition 1. Two nonempty subsets $A, B$ of a locally convex space $E$ are said to be Minkowski separated (resp., strongly Minkowski separated) if there exist a continuous Minkowski gauge $p$ on $E$ and a point $x_{0}$ in $E$ such that either $p(x)>$ $p(y)$ for all $x \in A_{x_{0}} \equiv A+x_{0}$ and $y \in B_{x_{0}} \equiv B+x_{0}$ or $p(x)<p(y)$ for all $x \in A_{x_{0}}$ and $y \in B_{x_{0}}$ (resp., either $\inf \left\{p(x) ; x \in A_{x_{0}}\right\}>\sup \left\{p(y) ; y \in B_{x_{0}}\right\}$ or $\left.\sup \left\{p(x) ; x \in A_{x_{0}}\right\}<\inf \left\{p(y) ; y \in B_{x_{0}}\right\}\right)$.

We replace the Minkowski gauge $p$ by a continuous linear functional in the above definition to obtain the common concept of separation sets. Clearly, two separated (resp., strongly separated) sets are Minkowski separation (resp., strong Minkowski separation) sets, if either of them is bounded.

Received by the editors December 12, 1994 and, in revised form, April 5, 1995.

1991 Mathematics Subject Classification. Primary 46A22.

Key words and phrases. Locally convex space, Banach space, Danes' Drop Theorem, separated sets.

(C)1996 American Mathematical Society 
Proposition 2. Suppose that $A$ is a convex set in a normed linear space $E$, which is a positive distance from $B \subset E$, if either of $A, B$ is bounded; then $A, B$ are strongly Minkowski separated.

Proof. Let $d=d(A, B)>0$ and let $S=\{x \in E ; d(A, x) \leq d / 2\}$. Then $S$, with nonempty interior, is a positive distance from $B$. Without loss of generality we assume that $0 \in \operatorname{int} S$ and let $P_{s}$ be the Minkowski gauge of $S$. It suffices to show

$$
R=\inf \left\{P_{s}(x)-P_{s}(y) ; x \in B, y \in S\right\}>0 .
$$

Note $d(S, B) \geq d / 2$.

Suppose, to the contrary, that $R=0$. Then we can choose sequences $\left\{x_{n}\right\},\left\{y_{n}\right\}$, from $B$ and $S$, resp., such that $P_{s}\left(x_{n}\right)-P_{s}\left(y_{n}\right) \rightarrow 0$; since $P_{s}\left(x_{n}\right)>1, P_{s}\left(y_{n}\right)<1$, we get $P_{s}\left(x_{n}\right) \rightarrow 1$. Let $k_{n}=P_{s}\left(x_{n}\right)^{-1}$; then $P_{s}\left(k_{n} x_{n}\right)=1, k_{n} x_{n} \in S$.

(i) If $B$ is bounded, then $\left\|x_{n}-k_{n} x_{n}\right\|=\left(1-k_{n}\right)\left\|x_{n}\right\| \rightarrow 0$. This contradicts the hypothesis that $d(S, B) \geq d / 2$.

(ii) If $A$ is bounded, then $S$ is bounded also. Thus, there exists a positive constant $k \geq 1$ such that $k^{-1} P_{s}(x) \leq\|x\| \leq k P_{s}(x)$ for all $x$ in $E$. Therefore $\left\{x_{n}\right\}$ is a bounded sequence. Hence

$$
\frac{d}{2} \leq d(S, B) \leq\left\|x_{n}-k_{n} x_{n}\right\| \rightarrow 0
$$

a contradiction which completes the proof.

\section{Danes' Drop Theorem in locally convex spaces}

Theorem 3. Given a sequentially closed bounded convex set $C$ in a sequentially complete locally convex space $(E, \tau)$. For every sequentially closed set $A$, which is strongly Minkowski separated from $C$, there exists a point $z \in A$ such that $D(z, C) \cap$ $A=\{z\}$, where $D(z, C)=\operatorname{co}(C \cup z)$ and co stands for the convex hull operator.

Proof. Without loss of generality we assume that $0 \in C$. Fix any $u_{0} \in A$. Let $G=\operatorname{s-clco}\left(C \cup-C \cup \pm u_{0}\right)$ (s-cl denotes $\tau$-sequential closure operator) and let $E_{1}=\operatorname{span} G$. Next, let $p$ be the Minkowski gauge by $G$; then it is a norm on $E_{1}$.

First, we show that $\left(E_{1}, p\right)$ is a Banach space. It suffices to show that the unit ball $G$ of $\left(E_{1}, p\right)$ is complete relative to $p$. Suppose that $\left\{x_{n}\right\}$ is a $\tau$-Cauchy sequence since $G$ is bounded and $p$ is generated by $G$, and which implies $\tau<\tau_{p}$ on $E_{1}$ where $\tau_{p}$ denotes the topology generated by the norm $p$. Since $C$ is $\tau$-sequentially complete, $x_{n}$ must be $\tau$-convergent to some point $x_{0}$ of $G$. Given a positive number $\varepsilon$, there is an integer $k$ such that $p\left(x_{m}-x_{n}\right)>\varepsilon$ whenever $m, n \geq k$, or equivalently $x_{m}-x_{n} \in \varepsilon G$, whenever $m, n \geq k$, because $G$ is $\tau$-sequentially closed, $x_{m}-x_{0} \in \varepsilon G$, that is, $P\left(x_{m}-x_{0}\right) \leq \varepsilon$ for all $m \geq k$. Therefore the sequence $\left\{x_{n}\right\}$ converges to $x_{0}$ relative to the norm topology $\tau_{p}$. Thus, $G$ is complete relative to $p$.

Since $C$ is bounded, convex and since $A$ is strongly Minkowski separated from $C$, Proposition 2 implies that there exist a point $x_{0} \in E$ and a $\tau$-continuous Minkowski gauge $p_{1}$ on $E_{1}$ such that

$$
p_{1}(x) \leq \alpha<\alpha+\varepsilon \leq p_{1}(y)
$$

whenever $x \in C+x_{0}, y \in A+x_{0}$ for some fixed $\alpha, \varepsilon>0$.

Without loss of generality we can assume that $x_{0}=0$ and write $(\varepsilon \leq) d=$ $\inf \left\{p_{1}(y)-p_{1}(x) ; x \in C, y \in A\right\}$. It is easy to see that $C$ is closed, bounded and 
convex relative to the norm $p_{1}$ of $E_{1}$ and $A \cap D\left(u_{0}, C\right)$ is also nonempty (it contains $u_{0}$, for example), closed and bounded. Now, define the function $f$ on $E_{1}$, by

$$
f(x)= \begin{cases}p_{1}(x), & x \in A \cap D\left(u_{0}, C\right), \\ \infty, & \text { otherwise }\end{cases}
$$

then $f$ is a norm $(p)$ lower semicontinuous proper function on $E_{1}$ since $p_{1}$ is $\tau$ continuous on $E$. Choose $\lambda>0$ such that diam $D\left(u_{0}, C\right)<d / \lambda$, where the diameter of $D\left(u_{0}, C\right)$ is in norm $p$. Use Ekeland's variational principle (see, for instance, [13] and [14]) to obtain a point $z \in A \cap D\left(u_{0}, C\right)$ such that

$$
f(x)+\lambda p(x-z)>f(z) \text { for all } x(\neq z) \text { in } E_{1} .
$$

We claim that $\{z\}=D(z, C) \cap A$. Suppose that $y \in D(z, C) \cap A$ with $y \neq z$. Then there exists $0<\mu<1$ and $v \in C$ such that $y=(1-\mu) z+\mu v$, so that $p_{1}(y) \leq(1-\mu) p_{1}(z)+\mu p_{1}(v)$ and $\mu d \leq \mu\left[p_{1}(z)-p_{1}(v)\right] \leq p_{1}(z)-p_{1}(y)$. Hence

$$
\begin{aligned}
p_{1}(z) & =f(z)<f(y)+\lambda p(y-z)=f(y)+\lambda p(\mu(v-z)) \\
& =p_{1}(y)+\lambda \mu(p(v-z)) \leq p_{1}(y)+\lambda \mu \operatorname{diam} D\left(u_{0}, C\right) \leq p_{1}(y)+\mu d \\
& \leq p_{1}(y)+\left(p_{1}(z)-p_{1}(y)\right)=p_{1}(z),
\end{aligned}
$$

a contradiction.

\section{ACKNOWLEDGMENT}

The authors wants to express their special thanks to Professor Wu Congxin for his helpful conversations on this note.

\section{REFERENCES}

1. J. Danes, A geometric theorem useful in nonlinear functional analysis, Boll. Un. Mat. Ital. 6 (1972), 369-372. MR 47:5678

2. - Equivalence of some geometric and related results of nonlinear functional analysis, Comment. Math. Univ. Carolinae 26 (1985), 443-454. MR 88a:47053

3. S. Rolewicz, On drop property, Studia Math. 85 (1986), 27-35. MR 88g:46033

4. J. R. Giles and Denka N. Kutzarova, Characterization of drop and weak drop properties for closed bounded convex sets, Bull. Austral. Math. Soc. 43 (1991), 377-385. MR 92d:46036

5. H. Brezis and F. E. Browder, A general principle on ordered sets in nonlinear functional analysis, Adv. in Math. 21 (1976), 355-364. MR 54:13641

6. F. Browder, Normal solvability and the Fredholm alternative for mappings into infinite dimensional manifolds, J. Funct. Anal. 8 (1971), 250-274. MR 44:5834

7. M. M. Day, Normed linear spaces, Springer, 1973. MR 49:9588

8. S. Dolecki and J. P. Penot, The Clark's tangent cone and limits of tangent cones, Publ. Math. Pau (1983), II-1-11.

9. M. Turcini, Mapping theorems via variable drops in Banach spaces, Rend. Istit. Lombardo, Sci. A 114 (1980), 164-168.

10. J. R. Giles, Brailey Sims and A. C. Yorke, On the drop and weak drop properties for a Banach spaces, Bull. Austral. Math. Soc. 41 (1990), 503-507. MR 91i:46014

11. Denka N. Kutzarova, On drop property of convex sets in Banach spaces, Constructive Theory of Functions' 87, Sofia (1988), 283-287.

12. D. N. Kutzarova and S. Rolewicz, On drop property for convex sets, Arch. Math. (Basel) $\mathbf{5 7}$ (1991), 385-394. MR 92i:46020

13. R. R. Phelps, Convex functions, monotone operators and differentiability, Lecture Notes in Math., vol. 1364, Springer-Verlag, 1989. MR 90g:46063 
14. J. R. Giles, Convex analysis with application in the differentiation of convex functions, Pitman Res. Notes in Math., vol. 58, 1982. MR 83g:46001

15. P. Georgiev, D. Kutzarova and A. Maaden, On the smooth drop property, Nonlinear Anal. 26 (1996), 595-602. CMP 96:03

Department of Mathematics, Jianghan Petroleum Institute, Hubei 434102, People's Republic of China

Current address: C. Lixin: Nankai Institute of Mathematics, Nankai University, Tianjin 300071, People's Republic of China 